\title{
Colza et apports azotés : enjeux d'une compétitivité durable et voies de recherche
}

\author{
Étienne PILORGÉ ${ }^{1}$ \\ Jacques EVRARD ${ }^{2}$ \\ Francis FLENET ${ }^{1}$ \\ Xavier PINOCHET ${ }^{1}$ \\ Raymond REAU ${ }^{3}$ \\ ${ }^{1}$ CETIOM, Centre de Grignon, BP4, \\ F-78850 Thiverval Grignon \\ ${ }^{2}$ CETIOM, rue Monge, Parcindustriel, \\ 33600 Pessac \\ ${ }^{3}$ INRA, INRA, UMR211, \\ INRA/AgroParisTech AGRONOMIE, \\ F-78850 Thiverval Grignon
}

La notion de développement durable s'est imposée en deux décennies, à partir du rapport Brundtland (1987), comme cadre incontournable d'une réflexion sur l'avenir. Dans ce cadre, penser la compétitivité d'une activité demande de s'interroger sur un ensemble de critères d'impacts et de les projeter dans l'avenir, en dépassant l'approche classique de la compétitivité économique à court et moyen terme, pour tendre vers une "compétitivité durable $^{1}$ ", dans une vision évidemment systémique, qui inclut non seulement la valeur des biens marchands, mais aussi ce qu'on appelle aujourd'hui des "externalités », positives ou négatives.

La production de colza s'insère dans plusieurs chaînes de valeur (huiles alimentaires, biodiesel, tourteaux, matière première pour la chimie verte... paysage et entretien du territoire) qui assurent leurs fonctions (nourrir différents usages) en impactant positivement et parfois négativement une série de domaines qui sont autant de lieux de compétitivité durable : envi-

\footnotetext{
$\overline{1}$ Traduction de «sustainable competitiveness »: «activités de croissance économique qui développent et restorent les systèmes naturels et sociaux desquels dépend la croissance économique présente et future, tout en, dans des conditions de marché équitables, produisant des biens et services adaptés aux marchés internationaux, et qui maintiennent ou augmentent le revenu réel de tous les citoyens » (W. MC Donough et M. Braungart).
}

\begin{abstract}
Nitrogen is a key factor in the sustainable competitiveness of oilseed rape crop and production systems, at the crossroads of economic and environmental stakes. The different research areas to meet these issues are examined, as well as their complementarities.
\end{abstract}

Key words: oilseed rape, nitrogen, sustainable competitiveness, cropping system

ronnement, société, consommateur, ressources énergétiques, économie des entreprises. Les impacts positifs deviennent d'ailleurs des fonctions à part entière dans la vision multifonctionnelle de l'agriculture, et les impacts négatifs les objets de progrès de la «cross compliance ».

\section{Quelle est la place de l'azote dans cette compétitivité durable?}

Parler de compétitivité suppose de se donner les moyens de la calculer ou de l'évaluer sur la base d'indicateurs.

Dans le domaine de l'économie des entreprises, le calcul est relativement simple et amène à identifier des bénéfices ou des pertes financières. Le poids d'un facteur comme l'azote se traduit directement en coûts de production, rendement, quantités produites et qualité.

Dans celui des ressources énergétiques, les bilans énergétiques et la méthode plus globale de l'analyse de cycle de vie (ACV) permettent d'évaluer les dépenses énergétiques des productions alimentaires et des productions de biocarburants. Là encore, l'évaluation du poids de l'azote est relativement aisée, à travers la consommation d'énergie pour sa fabrication et son transport.

Vis-à-vis de l'environnement, la mesure et l'évaluation des quantités d'effluents vers le sol, l'eau et l'air ont progressé ces dernières années.
Ces évaluations permettent de disposer de bases pour rentrer dans des dynamiques d'amélioration (limiter les pertes, absorber les excédents...) ou de valorisation (substituer des énergies renouvelables aux énergies fossiles par exemple). Mais sur le long terme, le simple bilan global des entrées et des sorties d'azote des systèmes de production ne suffit plus et la question se pose des seuils tolérables et des effets cumulatifs éventuels: évolutions des stocks d'azote et de carbone dans le sol, émissions vers les eaux (nitrates) ou l'atmosphère (ammoniac, protoxyde d'azote). La question du réchauffement climatique rend indispensable d'estimer la production de gaz à effet de serre, dont le protoxyde d'azote pour les activités agricoles et le méthane pour les activités d'élevage.

Vis-à-vis du consommateur, la compétitivité sociale peut s'évaluer principalement par la qualité et la sécurité sanitaire des produits (en comparaison de produits de substitution)... et bien-sûr par leur simple présence sur le marché en quantités suffisantes pour assurer les besoins des populations. L'azote est moins directement concerné par ces aspects de sécurité sanitaire et de qualité.

Au niveau social également, certains impacts de la production de colza restent évaluables comme ceux sur l'emploi. Le sujet devient plus complexe lorsqu'on aborde les questions de paysage, de qualité du cadre de vie, d'entretien du territoire et d'acceptabilité des pratiques. 
C'est sur ce dernier point que l'azote peut se trouver concerné, par ses impacts environnementaux et la consommation de ressources non renouvelable qu'il demande.

Si une partie des facettes de cette compétitivité durable est évaluable sans ambiguïté, une autre reste plus floue, du domaine de l'acceptabilité et du seuil de tolérance. Comme le suggère Jean-Christophe Kroll [1], une quatrième dimension de la durabilité apparaît « à côté de sa dimension économique, sociale et environnementale : celle de son acceptabilité politique ", dont on voit l'importance sur le cas des biocarburants et en particulier des pratiques de fertilisation azotée.

Il convient de constater que la pratique agricole de la fertilisation azotée du colza, et son raisonnement, se trouvent, parmi d'autres éléments, au confluent de plusieurs grands enjeux au cœur des politiques européennes :

- la compétitivité des entreprises agricoles, par la maîtrise des coûts, et donc l'activité économique et l'occupation des espaces ruraux

- la pratique d'une agriculture respectueuse de I'environnement, et la baisse du risque de fuites de nitrates vers les eaux : écoconditionnalité des aides agricoles, politiques de l'environnement ;

- la limitation des émissions de gaz à effets de serre, pour le respect des engagements de I'UE dans le cadre des accords de Kyoto : d'une part par l'amélioration du bilan énergétique des cultures de colza biodiesel, qui fait écho à la Stratégie européenne pour les biocarburants, et d'autre part par la limitation des émissions de protoxyde d'azote des cultures de colza en général.

Le sujet de l'azote est complexe dans la mesure où il intervient non seulement dans des questions de compétitivité simple, «facilement » objectivables, mais aussi de compétitivité de long terme, lieu de débats où la dimension du politiquement soutenable devient prédominante.

II est également complexe car le traitement des problèmes doit être abordé, et les progrès réalisés, à différentes échelles spatiales et temporelles qui constituent autant de sous-systèmes emboîtés : de la plante considérée dans son métabolisme et dans ses relations physiologiques avec le sol et le climat, au peuplement végétal de la parcelle agricole (échelle spatiale de la parcelle et temporelle de l'année), puis à la rotation culturale (parcelle à l'échelle pluriannuelle) et au système de production (échelle de l'exploitation agricole), voir au bassin de production et au bassin-versant.

Les échelles de consolidation des principaux impacts vont de la parcelle (rendement) au bassin-versant (charge des eaux de surface en nitrates) et à la planète (gaz à effet de serre). Et la pertinence et la réalité des progrès réalisés à l'une de ces échelles doivent être considérées à I'aune des impacts (progrès complémentaires induits ou régressions) aux autres échelles (tableau 1).

\section{Compétitivité économique et qualité des produits}

Le respect des objectifs de la filière biodiese pour répondre aux politiques européenne et nationale sur les biocarburants (objectifs fixés par le gouvernement français de $5,75 \%$ en $2008,7 \%$ en 2010 et $10 \%$ en 2015 ) sans hypothéquer les besoins alimentaires, nécessite d'assurer un approvisionnement croissant et durable en huile, principalement de colza. Cet engagement requiert une augmentation des surfaces, et donc un intérêt économique accru pour les producteurs, mais également une amélioration du rendement huile par hectare. Malgré une tendance à la baisse des quantités moyennes d'engrais de synthèse apportées de l'ordre de $8 \%$ sur les cinq dernières années (passant de 181 kg d'azote par hectare en 1999 à 167 en 2005), due en grande partie à la diffusion croissante de la méthode de fertilisation raisonnée par la réglette azote colza du Cétiom, la fertilisation azotée était en 2005 le premier poste de charge et représentait $29 \%$ des charges opérationnelles en moyenne (source : enquêtes Cétiom). Les coûts de production du colza sont donc assez sensibles aux évolutions des prix des engrais azotés, euxmêmes fortement dépendants du prix de l'énergie: le gaz naturel représente environ $50 \%$ du prix de vente des engrais azotés, lequel a augmenté de plus de $35 \%$ entre juin 2000 et juin 2006.

\section{Amélioration de l'utilisation apparente de l'azote par le colza par la conduite de culture}

Sur le court et moyen terme, réduire les impacts de cette hausse passe par la valorisation de l'azote déjà présent dans le sol et l'amélioration de l'utilisation apparente de l'engrais (réduction des quantités apportées à rendement égal), et/ou par le recours à des sources bon marché.

L'exploitation de l'azote présent dans le sol sous forme assimilable, et plus particulièrement I'utilisation apparente de I'azote de I'engrais par le colza est normalement relativement élevée, grâce à sa croissance rapide à l'automne et à son long cycle de développement.

En premier lieu, assurer une levée relativement précoce permettra que l'azote minéral disponible dans le sol en automne soit complètement assimilé par le colza avant l'hiver, une période où le drainage pourrait entrainer les nitrates au-delà de la zone racinaire. Puis il faudra évi- ter, par une conduite de culture adaptée, de faire chuter les coefficients d'utilisations. L'adaptation de la conduite de la culture, et notamment des pratiques de fertilisation, devrait permettre $d^{\prime}$ atteindre régulièrement des CAU de plus de $80 \%$.

Le premier niveau d'action consiste évidemment à éviter la surfertilisation, et donc de progresser sur le raisonnement de la fertilisation, sujet par nature très intégrateur. Les principes de base du raisonnement restent ceux de la méthode du bilan. De ce point de vue, une première source de progrès est intimement dépendante de l'amélioration des connaissances et des références sur les différents postes du bilan appliqués à la culture de colza : quantités d'azote mobilisées par la culture pour élaborer son rendement, potentiels de minéralisation des différents types de sols sous différents climats et systèmes de culture, etc. Dans le cas particulier du colza, l'introduction des quantités d'azote absorbées par la culture en fin d'hiver dans la démarche classique de la méthode du bilan, qui a fait l'objet de travaux au début des années 1990 et d'une déclinaison en développement quelques années plus tard, a fortement contribué aux progrès mentionnés ci-dessus, sans affecter le rendement graines.

La limite de cette méthode réside dans le fait d'apporter une dose moyenne à l'échelle de la parcelle : un progrès est encore possible grâce à une modulation dans l'espace de la parcelle cultivée: depuis quelques années, l'élaboration de cartes de biomasse à partir de photographies satellites dans le cadre du projet Farmstar permet non seulement de proposer des doses d'apport moyennes, mais permet aux agriculteurs d'adapter les doses en fonction des hétérogénéités spatiales de la culture. La variabilité entre zones d'une même parcelle peut en effet être importante et du même ordre de grandeur que la variabilité entre des parcelles différentes : de 40 à plus de 150 unités d'azote absorbées par la culture en sortie d'hiver, cette valeur étant la donnée d'entrée pour l'application de la méthode de la réglette colza dérivée de la méthode des bilans. Certains agriculteurs ont mis en pratique la modulation intraparcellaire de la fertilisation azotée sans matériel spécifique, par grandes zones homogènes, d'autres, encore peu nombreux, allant jusqu'à une modulation à l'aide de matériel d'agriculture de précision utilisant les cartes numérisées. Des économies substantielles sont réalisables par cette technique.

À cette modulation dans l'espace répond une modulation dans le temps, autre voie importante de progrès et d'amélioration de l'utilisation apparente de l'azote de l'engrais: en apportant l'engrais de façon fractionnée et en période de forte croissance, en évitant les apports quand le sol est encore bien pourvu en 


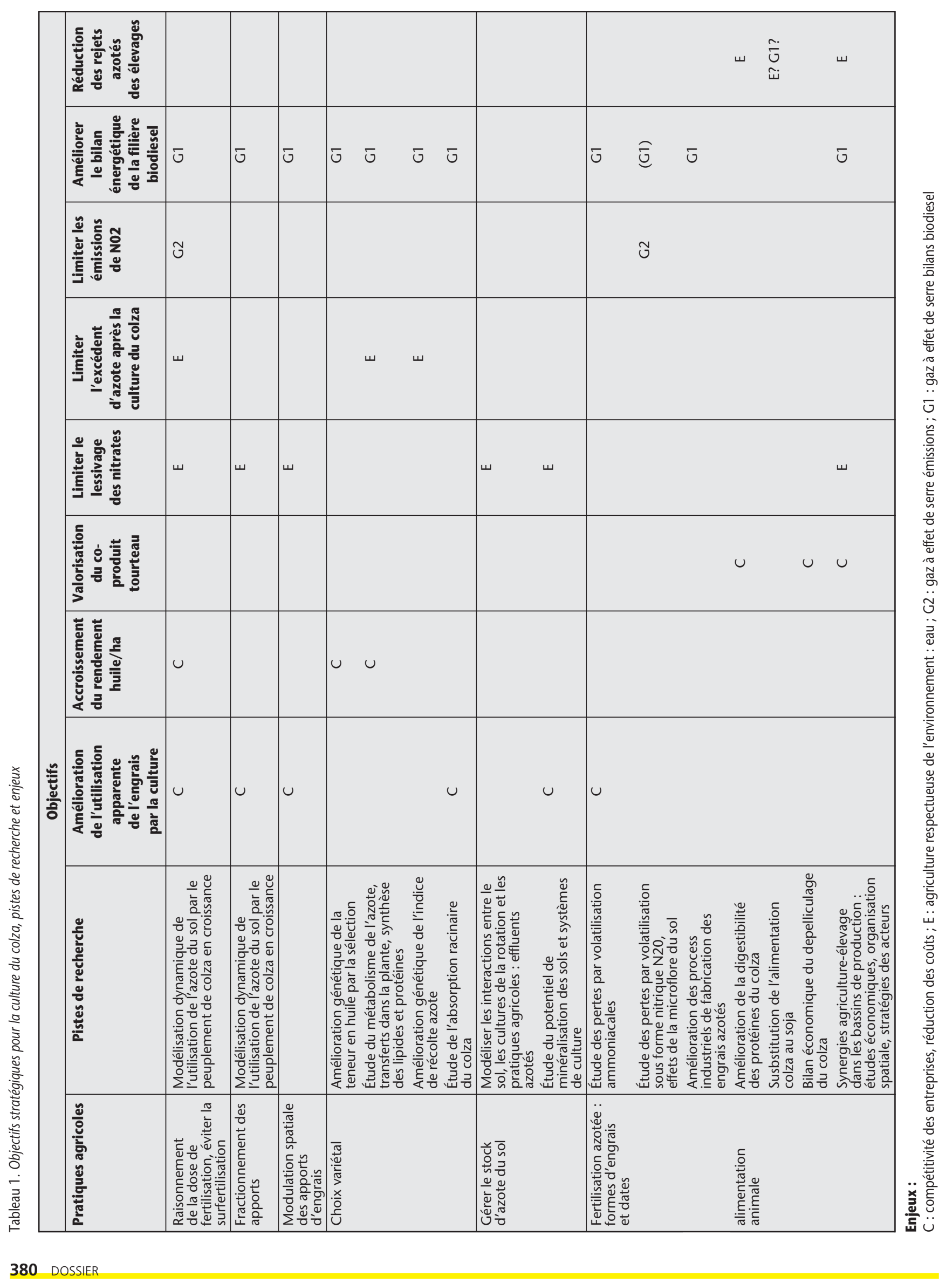


azote minéral, ou les apports « pour compenser » de faibles conditions de croissance de fin d'automne. La modélisation de l'absorption de l'azote du sol par le peuplement de colza en croissance, en interaction avec les conditions météorologiques et les pratiques agricoles, est une voie de recherche privilégiée pour concevoir des outils de diagnostic et de raisonnement des doses et du fractionnement des apports d'azote dans le temps. Des travaux sont en cours dans le cadre de l'adaptation du modèle dynamique Azodyn au colza pour le raisonnement de la fertilisation azotée, et du projet Azosystem pour le diagnostic des pertes dans le système de culture (voir plus bas).

Enfin, à plus long terme, au-delà de I'amélioration de l'utilisation de l'azote, il est possible d'imaginer améliorer la compétitivité économique du colza par la réduction de ses besoins en azote : un rendement et une qualité au moins équivalents avec une moindre mobilisation d'azote, c'est-à-dire une amélioration de l'indice de récolte azote par diminution de I'azote contenu dans les résidus, dont nous reparlons plus tard.

\section{Optimisation du produit brut : rendement huile et valorisation des tourteaux}

Faut-il le rappeler, le colza est d'abord un oléagineux, dont la valorisation provient principalement de l'huile, à tel point que la valeur de la seule fraction huile issue de trituration est proche de celle de la graine brute, aux cours actuels des produits. Compte tenu du contexte de demande en huile, c'est bien le rendement huile qu'il faut optimiser, dans l'intérêt de I'ensemble des acteurs de la filière. La teneur en huile est un facteur de compétitivité comme le rendement. Or pour une variété donnée, la surfertilisation azotée accroît la teneur en protéines des graines et diminue la teneur en huile. L'accroissement du rendement huile par la teneur en huile des graines récoltées suppose d'agir à deux niveaux :

- sur la conduite de culture, en évitant la surfertilisation (baisse d'un demi point d'huile pour un surplus de $45 \mathrm{~kg}$ d'azote/ha) : il s'agit d'optimiser le rendement huile, et pas seulement le rendement graines, vis-à-vis du facteur azote ;

- sur l'amélioration génétique de la teneur en huile par la sélection: les réels progrès en matière d'amélioration génétique doivent être poursuivis.

Néanmoins, la fraction tourteau de la graine contribue aussi à sa valorisation, et la question devient d'autant plus cruciale que s'accroissent les quantités disponibles, en co-produit de la filière biodiesel. Malheureusement, pour une variété donnée la teneur en protéines de la graine est corrélée négativement à la teneur en huile et bien que la relation ne soit pas stricte compte tenu des parois de la graine, le risque existe d'une dégradation de la teneur en protéines du tourteau au fil de l'amélioration de la teneur en huile de la graine.

Ces questionnements doivent conduire à examiner les interactions entre métabolisme de I'azote et synthèse lipidique, peu explorés à ce jour, en complément des connaissances sur la synthèse des protéines. Des travaux de recherche conjointe entre I'Inra EGC Grignon et l'équipe Écophysiologie végétale de l'université de Caen, sont menés actuellement sur les questions de partition des ressources carbonées. II s'agit d'identifier les mécanismes susceptibles d'augmenter la remobilisation de l'azote foliaire vers les tissus reproducteurs en identifiant leur déterminisme environnemental (dont la disponibilité en azote) et génétique. Ces travaux doivent déboucher sur la mise au point de modèles à compartiments et d'un modèle architectural, qui doit permettre de prendre en compte les processus à l'échelle des organes et de tester leur sensibilité génotypique aux conditions environnementales.

Au niveau de la graine récoltée, l'objectif principal reste bien l'augmentation de la teneur en huile, plusieurs éléments permettant d'accepter un statu quo sur les protéines. D'une part, si I'amélioration de la digestibilité des nutriments du tourteau de colza (énergie et protéines) aurait un fort impact sur l'augmentation de son prix d'intérêt dans les formules pour monogastriques, il apparaît qu'une augmentation de la teneur en protéines du tourteau en tant que telle a peu d'influence sur son prix d'intérêt dans les formules porcs (sauf indirectement via une augmentation de sa teneur en acides aminés digestibles). C'est donc plus une amélioration de la digestibilité des protéines du colza qu'il faut viser en priorité. Par ailleurs, la voie technologique du dépelliculage existe toujours, qui permettrait d'augmenter la teneur en protéines des tourteaux. Le bilan économique du dépelliculage, incluant la valorisation des pellicules (et de leur teneur en huile résiduelle) dans les chaudières des unités de trituration, mériterait d'être réexaminé.

Par ailleurs, il est important de souligner que la digestibilité des protéines relève également $d^{\prime} u n$ enjeu environnemental : la réduction des pertes en azote par les effluents d'élevages, point particulièrement critique dans les régions d'élevages hors sol. II s'agit là d'une autre interaction dans laquelle l'azote du colza est impliqué, d'intérêt non négligeable pour la compétitivité environnementale des systèmes de production en régions de polyculture-élevage.

\section{Durabilité environnementale : réduction des impacts sur les ressources naturelles, air, eau}

L'azote joue un rôle important dans la compétitivité environnementale du colza.

Le colza est une culture paradoxale dans la mesure où le solde entre les apports d'azote sur la parcelle cultivée, par la fertilisation, et les exportations par la culture (I'azote contenu dans les graines) est relativement élevé, de l'ordre de $80 \mathrm{~kg}$ d'azote par hectare, supérieur à celui du blé alors que pour autant, les fuites d'azote hors de la parcelle ne semblent pas s'accroître lorsqu'on introduit un colza dans une rotation céréalière. Ce solde traduit en partie l'effet précédent du colza qui laisse un peu plus d'azote dans le sol à la récolte, mais il intègre aussi sans l'identifier le rôle de piège à nitrate du colza au cours de l'automne suivant son semis. Une autre hypothèse expliquerait ce comportement : le colza pourrait contribuer à améliorer le stock de matière organique du sol sous forme d'humus, et donc parallèlement à stocker de l'azote et du carbone dans le sol, ce qui serait un atout vis-à-vis de l'effet de serre. Vérifier ces hypothèses permettrait peut-être de mieux exploiter ces mécanismes, si leur réalité se confirme.

Une culture de colza bien conduite présente l'avantage de couvrir le sol une grande partie de l'année, et, compte tenu de ses fortes capacités d'absorption, de limiter les pertes d'azote vers les eaux. Les risques de perte d'azote les plus importants dans les systèmes de culture avec colza sont principalement :

- sous forme de nitrates vers les eaux, principalement après le colza avant l'implantation du blé et pendant l'hiver alors que les capacités d'absorption du blé restent limitées et les risques de lessivage élevés ;

- sous forme ammoniacale $\mathrm{NH} 3$, par volatilisation dans les jours qui suivent les apports de fertilisants ammoniacaux ;

- sous forme de protoxyde d'azote N2O quand le sol est riche en azote sous forme de nitrates en surface, particulièrement en présence d'eau libre et de chaleur.

\section{Limiter le lessivage des nitrates}

Pour limiter le lessivage des nitrates, l'attention doit être portée sur la gestion du stock d'azote de la parcelle, notamment après le colza. II peut en effet laisser des quantités d'azote minéral relativement plus élevées dans le sol, du fait qu'il cesse d'absorber relativement tôt, que ses résidus relativement riches en azote et pauvres en carbone réorganisent moins d'azote minéral, et que des phénomènes de minéralisation estivale restent possibles. Enfouir les résidus de culture pendant l'été permet d'optimiser 
I'organisation de l'azote minéral et les repousses spontanées, qu'il convient de favoriser, permettent de "piéger » ces quantités d'azote sous forme de nitrates présentes dans le sol après la récolte du colza, et de réduire de façon très significative les pertes dans la rotation, ce qui devrait permettre de réduire les apports d'azote moyens à la rotation.

La gestion du stock d'azote au cours de la rotation reste délicate et demande la mise au point d'outils de diagnostic des risques de pertes et de pilotage de la fertilisation. La difficulté est en effet de comprendre, de façon suffisamment précise pour les gérer, les interactions entre le sol, les conditions climatiques, les cultures en place au cours de la rotation et les pratiques agricoles dont elles sont l'objet. II convient donc de développer à la fois des modèles dynamiques et des outils de diagnostic des pertes d'azote au cours de la rotation à l'échelle de la parcelle agricole. Ce dernier point fait l'objet du projet Azosystem.

Les outils de pilotage de la fertilisation et du stock d'azote du sol devront évoluer en parallèle pour valoriser ces acquis, et s'appuyer sur des connaissances complémentaires des mécanismes et des interactions, ainsi que sur des références adaptées aux différentes régions de culture, notamment sur les aspects types de sols et systèmes de culture : de nouveaux travaux sur le potentiel de minéralisation des sols sont en cours, qui ont fait l'objet d'une thèse à I'Inra Toulouse, cofinancée entre le Cétiom et Arvalis-Institut-du-Végétal [2] et contribuent à élargir les acquis des équipes INRA de Laon.

\section{Des résidus de culture de colza: pas assez pauvres en azote}

Les résidus du colza méritent une attention particulière dans la mesure où ils sont relativement riches en azote, avec des rapports $\mathrm{C} / \mathrm{N}$ de l'ordre de 50 quand ceux des pailles de céréales dépassent 100 . Ces résidus sont constitués de feuilles qui tombent au sol avant la maturité, et de tiges et de parois de siliques qui se décomposent dans le sol après la récolte. Leurs caractéristiques font que le sol est plus riche en azote minéral après un colza $(+10 \mathrm{~kg}$ à la récolte, $+40 \mathrm{~kg}$ en sortie d'hiver), ce qui contribue certainement à la réputation de bon précédent qui est celle du colza.

Compte tenu de ces caractéristiques, les résidus de colza organisent l'azote minéral du sol, mais moins que les pailles de céréales, ce qui explique aussi qu'on trouve plus d'azote minéral dans le sol après colza.

Les variétés naines, si elles permettent de réduire les besoins en azote ( $\mathrm{N}$ absorbé/quintal), présentent peu d'intérêt du point de vue de la limitation du lessivage des nitrates si les résidus en moindre quantité (réorganisation moins importante) restent riches en azote.

Une voie de progrès résiderait donc dans des résidus de culture moins riches en azote. La maîtrise de la fertilisation peut y contribuer, mais la marge de manœuvre est limitée, à rendements équivalents.

L'amélioration génétique peut probablement contribuer à cet objectif dans la mesure où il existe une variabilité génétique de l'indice de récolte azote du colza (IRN) comme l'ont montré au niveau exploratoire les travaux engagés dans le cadre des projets IRN, puis Natural. L'objectif serait d'augmenter cet IRN (rapport (quantité d'azote contenue dans les graines/ (quantité d'azote contenue dans les graines + quantité contenue dans les « résidus »)), en diminuant l'azote contenu dans les résidus et, éventuellement en augmentant l'azote contenu dans les graines.

En appui aux travaux de génétique, il est nécessaire de mieux comprendre le métabolisme de l'azote et des transferts au cours de la vie des plantes, leurs effets sur la répartition de l'azote dans la plante, et l'élaboration des rendements huile et protéines. Développer des travaux de génétique sur ces aspects demande au préalable que la ou les cibles soient bien identifiées, ce qui nécessite de comprendre les interactions entre remobilisation, sénescence et stockage de l'azote dans la plante sur la seconde partie du cycle, et ramène aux travaux des équipes Inra EGC Grignon et de I'Université de Caen, cités plus haut.

\section{Limiter les fuites par volatilisation : $\mathrm{N} 2 \mathrm{O}, \mathrm{NH} 3$}

Limiter les pertes d'azote par volatilisation relève à la fois de l'enjeu économique et de la limitation des gaz à effet de serre, qui relève de l'environnement et de l'acceptabilité politique. La majeure partie des pertes d'azote par volatilisation est sous forme ammoniacale (NH3). Les pertes varient en fonction de la molécule apportée, de la forme de l'engrais (solide/liquide), du temps de transformation nécessaire pour parvenir à la forme nitrate assimilable, lui-même conditionné par le climat avant apport (humidité du sol) et après apport (température et pluviométrie). L'ordre de grandeur des pertes potentielles par volatilisation de l'ammoniac des engrais minéraux dans les situations favorables au phénomène pourrait atteindre plus de $20 \%$ de la quantité épandue, l'essentiel des pertes intervenant dans les 48 heures suivant l'apport. Les conditions favorisant la volatilisation ammoniacale résident dans les sols calcaires ou à $\mathrm{pH}$ élevés, les températures de l'air élevées $\left(20-25^{\circ} \mathrm{C}\right)$ et les conditions venteuses. L'humidité du sol semble jouer de façon plus complexe, et la forme d'engrais elle-même interagit avec les condi- tions météorologiques. Les connaissances sur le sujet, dans le cas de la culture du colza en particulier, restent limitées et mériteraient d'être approfondies. Néanmoins, éviter les apports d'azote par temps sec ou chaud ou venteux constituerait une précaution de base, a fortiori sur terres calcaires.

Les pertes sous forme de protoxyde d'azote semblent beaucoup moins importantes en quantités, de l'ordre de $1 \%$, mais leur enjeu réside dans l'impact élevé de ce gaz sur l'effet de serre, 296 fois plus élevé que celui de la référence $\mathrm{CO} 2$, à masse identique. On considère actuellement que le protoxyde d'azote est responsable de $5 \%$ de l'effet de serre, et qu'un tiers de ses émissions proviennent de l'activité agricole. Cette caractéristique et les remises en cause des cultures de colza à usage biocarburant par les milieux environnementalistes ont conduit à une série de recherches sur le sujet, permettant d'estimer les émissions réelles des parcelles de colza, et à l'analyse du cycle de vie du colza sur les aspects de bilans énergétiques et de bilans en émissions de gaz à effet de serre. Les études ont montré que les émissions de N2O, en moyenne de l'ordre de 1,2 kg/ha par an, étaient très variables, et dépendaient notamment des types de sols, les sols riches ou à tendance hydromorphe présentant les émissions les plus élevées.

Les recherches menées par I'Inra et le Cétiom ont montré une tendance identique sur les différents sites étudiés : les émissions de protoxyde d'azote s'accroissent lorsqu'on passe d'un couvert végétal non fertilisé à un sol nu, puis à un colza normalement fertilisé et enfin à un colza surfertilisé.

Le potentiel de dénitrification d'un sol dépend des quantités d'azote nitrique en surface, des conditions hydriques, de la température et des quantités et formes du carbone du sol. Les sols de limons lessivés qui cumulent des niveaux de carbone organique élevés et des périodes d'anoxie plus longues et plus fréquentes liées à la présence d'une couche d'argile sous-jacente, constituent un type de situation à risque. Ce risque peut être diminué par le drainage. II apparaît clairement que la surfertilisation augmente les émissions de $\mathrm{N} 2 \mathrm{O}$, et les résultats suggèrent que cette augmentation serait plus que proportionnelle à la disponibilité en nitrates dans I'horizon de surface. « La relation entre le niveau d'azote nitrique disponible et l'intensité des émissions de $\mathrm{N} 2 \mathrm{O}$ conduit à penser que les techniques destinées à éviter les fortes concentrations d'azote minéral dans le sol, notamment en période pluvieuse, telles que l'ajustement strict des apports aux besoins, le fractionnement des apports azotés, I'utilisation des repousses comme culture intermédiaire piège à nitrate, devraient réduire ces risques d'émission. » [3]. Les travaux sur le sujet restent 
à poursuivre, notamment sur les effets de l'activité de la microflore du sol, dont les variations pourraient expliquer des pics d'émissions et le contrôle contribuer à les limiter.

\section{Compétitivité de long terme, durabilité des systèmes de production et recevabilité sociopolitique}

\section{Diminution du poids de l'azote dans les bilans énergétiques}

La recevabilité sociopolitique de la production de biocarburants est clairement liée aux économies d'énergie fossile qu'ils permettent et à leur contribution à la limitation des émissions de gaz à effet de serre. Si la question des émissions de N2O mérite d'être examinée en tant que telle, celle du bilan CO2 est intimement liée au bilan énergétique de la filière de production de biodiesel, dont l'amélioration est donc essentielle.

À ce niveau, l'azote intervient aussi de façon déterminante du fait de son « contenu énergétique » : l'énergie mobilisée pour la fabrication et le transport de l'engrais azoté est le premier poste de dépense énergétique de la culture du colza et représente environ $48 \%$ de la dépense totale, devant la mécanisation (40\%) [4].

Les voies de progrès se situent là aussi à plusieurs niveaux.

L'amélioration des process industriels de fabrication de l'engrais azoté et l'application des technologies les plus performantes devraient permettre une baisse de 15 à $20 \%$ de la quantité d'énergie utilisée selon la forme d'engrais fabriquée, par rapport aux performances moyennes européennes [5].

À moyen terme, la poursuite de l'extension du travail du sol simplifié, sans labour, peut également contribuer de façon significative à la réduction des coûts énergétiques de la culture, mais elle doit être accompagnée de progrès parallèles en matière de contrôle des adventices et de certaines maladies (gestion des résidus des cultures).
Sur le long terme, c'est cette forte dépendance de la culture qu'il conviendrait de diminuer pour obtenir un colza plus efficient dans I'utilisation de l'azote, ressource qui risque fort de se raréfier : nous retrouvons là la problématique de l'amélioration de l'indice de récolte azote envisagée plus haut.

Sur le long terme également, une meilleure compréhension du fonctionnement de l'absorption racinaire (durée de fonctionnement, régulations, taille du système racinaire) permet d'envisager une meilleure exploitation de l'azote présent dans le sol et une amélioration des coefficients d'utilisation de l'engrais azoté. II s'agit là de travaux de physiologie, de génétique, sur les auxiliaires biologiques (bactéries rhizosphériques) et les interactions avec le travail du sol.

\section{Développement plus intégré dans les régions d'élevage: réduction globale des impacts}

Sur le long terme, les enjeux se rejoignent : la durabilité des systèmes de production nécessite une performance économique, environnementale et sociopolitique. Considérer l'échelle du système de production permet d'envisager $d$ 'autres voies de progrès, en particulier quant aux synergies possibles entre systèmes de production agricoles demandeurs d'azote minéral et organique, et systèmes d'élevage utilisateurs de produits protéiques et émetteurs d'effluents azotés par les déjections animales.

Deux voies de synergies méritent d'être explorées et mises en valeur : d'une part l'étude des potentialités de limitation des rejets azotés des élevages offertes par le remplacement du soja par des tourteaux de colza dans l'alimentation du bétail. Un point faible actuel du colza réside dans la digestibilité des protéines du tourteau de colza, qui est assez moyenne et dont la mauvaise utilisation peut être source de rejets, malgré la plus faible concentration protéique des rations à base de colza. L'amélioration de la digestibilité des protéines du colza est donc une voie à privilégier. Par ailleurs, les émissions de méthane par les bovins peuvent varier en fonction de leur alimentation : les caractéristi- ques de l'alimentation colza de ce point de vue mériteraient d'être examinées, à l'exemple d'une préétude réalisée pour le lin.

D'autre part, l'utilisation de colza produit localement permettrait de limiter le caractère excédentaire du bilan azoté d'un bassin de polyculture-élevage, par limitation des importations d'azote sous forme de tourteau de soja. Des synergies pourraient probablement être développées entre exploitations d'élevage et exploitations de culture à l'échelle de petites régions. Une première catégorie de besoins de recherche porte sur l'organisation et l'économie des systèmes de production, de collecte et de transformation, et sur les stratégies des acteurs, en vue de définir les synergies exploitables.

Le développement du colza énergétique dans les régions d'élevage en excédent structurel permettrait de valoriser son rôle de pompe à nitrate, en particulier sur la période automnehiver, et de produire des colzas sans fertilisation azotée minérale, améliorant fortement le bilan énergétique de la culture tout en contribuant à la limitation des pollutions.

Enfin, le pilotage des améliorations aux différentes échelles d'action envisagées demande de disposer d'indicateurs pour les différentes composantes de la compétitivité durable.

\section{RÉFÉRENCES}

1. KROLL JC. Les politiques actuelles permettentelles d'atteindre un développement durable en agriculture? OCL 2006 ; 13(2-3) : 90-102.

2. VALE M. Quantification et prédiction de la minéralisation nette de l'azote du sol in situ, sous divers pédoclimats et systèmes de culture français. 2006.

3. GERMON JC, HENAULT C, PAGE S, LUCAS JL, REAU R. Les émissions de protoxyde d'azote sous culture de colza. OCL 1998 ; 5(1) : 5-11.

4. ADEME, DIREM. Bilans énergétiques et gaz à effet de serre des filières de production de biocarburants. Rapport technique Ecobilan PriceWaterhouseCoopers, Novembre 2002, ADEME, DIREM.

5. La Lettre de UNIFA N 13, septembre 2005. 